\section{IDDF2020-ABS-0119 DISSECTING THE MOLECULAR MECHANISM AND CLINICAL SIGNIFICANCE FOR REGULATING THE MALIGNANT PROGRESSION OF GASTRIC CANCER BY HIPK3}

Xiao-Jing Luo*, Qi-Nian Wu, Ze-Xian Liu, Rui-Hua Xu. Sun Yat-Sen University Cancer Center, China

\subsection{6/gutjnl-2020-IDDF.26}

Background Gastric cancer (GC) is one of the most threatening malignant diseases in east Asia with largely unknown mechanisms. Protein kinases and their signaling pathways were closely related to cancer occurrence and progression. We decide to investigate the role of related protein kinases and their signaling pathways in GC.

Methods We analyze the protein kinases activity using the phosphorylated proteomic data of gastric cancer and find that the substrates of HIPK family with abnormal phosphorylated levels. HIPK3 with less known function and mechanisms is chosen for further research, and its low expression in GC is confirmed by qPCR and immunohistochemistry analysis in a large GC patient cohort. MTS assay, colony formation assay, transwell migration and invasion assays are performed after ectopic express or knockdown of HIPK3 in vitro. Additionally, subcutaneous xenograft and in situ xenograft models are built in vivo. We detect the HIPK3 mRNA and protein level in cisplatin-resistant GC cells by qPCR and western blotting and measure the tolerance of cisplatin after overexpression of HIPK3. Coimmunopreicipitation and mass spectrometry analysis identify the interaction between HIPK3 and MAP7. Immunofluorescence confirms their co-localization. Downstream mechanisms were examined by regular molecular biological methods.

Results We discovered the aberrant phosphorylation of substrates for HIPK family kinases based on the profiled gastric cancer phosphoproteome data and bioinformatical pipeline for kinase activity analysis. Then the low expression of HIPK3 in the gastric tumor was validated and correlated with patient overall survival. Cell line and mouse model experiments for gastric cancer showed that reducing the HIPK3 expression promoted the growth, proliferation, migration, invasion and metastasis, and high expression of HIPK3 could reverse the cisplatin resistance. Further molecular mechanism revealed that HIPK3 might regulate the progression of gastric cancer through phosphorylating MSH6 to regulate DNA mismatch repair and interaction with MAP7 to regulate cell morphology.

Conclusions Our study explicit the potential of HIPK3 as a key regulator for progression, an effective biomarker for prognosis and drug resistance, and a potential therapy to target gastric cancer.

\section{IDDF2020-ABS-0157 THERAPEUTIC EFFECT OF DIETARY N-3 POLYUNSATURATED FATTY ACID IN CROHN'S DISEASE BY RESTORING TH17/ TREG IMBALANCE THROUGH PPAR-R/ AMPK/ACC MEDIATED PATHWAY}

${ }^{1}$ Jiayin Yao*, ${ }^{2}$ Bang Hu, ${ }^{3}$ Jiachen Sun, ${ }^{1}$ Min Zhi. ${ }^{1}$ Department of Gastroenterology, Guangdong Provincial Key Laboratory of Colorectal and Pelvic Floor Disease, The Sixth Affiliated Hospital of Sun Yat-Sen University, China; ${ }^{2}$ Department of Colorectal Surgery, The Sixth Affiliated Hospital of Sun Yat-Sen University, China; ${ }^{3}$ Department of Gastrointestinal Endoscopy, The Sixth Affiliated Hospital of Sun Yat-Sen University, China

\subsection{6/gutjnl-2020-IDDF.27}

Background Effective treatments of Crohn's disease (CD) are limited. Diet is not only an environmental factor in the pathogenesis of CD but also an underlying therapeutic target. Our previous study confirmed the protective effect of dietary n-3 polyunsaturated fatty acid (n3-PUFA) in CD rats without mechanisms explored in-depth. In this study, we aimed to investigate and validate the mechanisms of $n-3$ PUFA in CD in vivo and vitro focusing in $\mathrm{T}$ cell differentiation and immune disorder.

Methods Experimental CD-like colitis rats were induced by 2,4,6-trinitrobenzene sulfonic acid (TNBS) and naïve $\mathrm{T}$ cells were isolated from colonic mucosa lamina propria. Disease

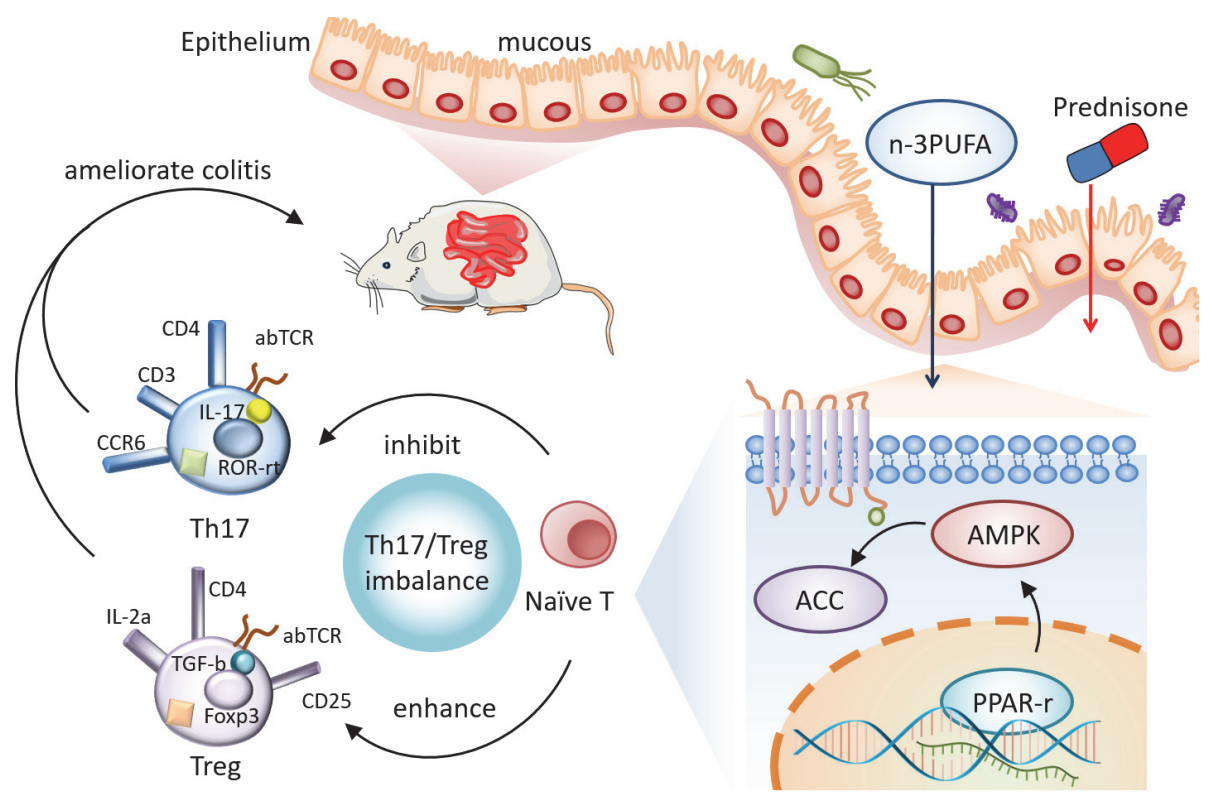

Abstract IDDF2020-ABS-0157 Figure 1 Hypothesis chart of this study 